1 Running title: No independent division machinery in thylakoids

2

3

4

5

6

7
Corresponding author details: Steven M. Theg, Department of Plant Biology, University of California Davis, Davis, CA 95616

\section{Characterization of Thylakoid Division Using Chloroplast Dividing Mutants in Arabidopsis}

\author{
Jonathan Ho, Warren Kwan, Vivian Li, and Steven M. Theg ${ }^{1}$ \\ Department of Plant Biology, University of California Davis, Davis, CA, 95616
}

Summary: Chloroplasts unable to divide possess giant thylakoids suggesting that thylakoids do not possess independent division machinery

\footnotetext{
Author Contributions: JH and SMT designed experiments. JH, WK and VL performed the experiments and all authors analyzed the data. JH and SMT wrote the article and all authors approved it.

Funding information: This work was funded by the Division of Chemical Sciences, Geosciences, and Biosciences, Office of Basic Energy Sciences of the US Department of Energy through Grant DE-FG02-03ER15405.

${ }^{1}$ Address correspondence to smtheg@ucdavis.edu.
} 


\section{Abstract:}

25 Chloroplasts are double membrane bound organelles that are found in plants and algae. Their

26 division requires a number of proteins to assemble into rings along the center of the organelle

27 and to constrict in synchrony. Chloroplasts possess a third membrane system, the thylakoids,

28 which house the majority of proteins responsible for the light-dependent reactions. The

29 mechanism that allows chloroplasts to sort out and separate the intricate thylakoid membrane

30 structures during organelle division remain unknown. By characterizing the sizes of thylakoids

31 found in a number of different chloroplast division mutants in Arabidopsis, we show that

32 thylakoids do not divide independently of the chloroplast division cycle. More specifically, we

33 show that thylakoid division requires the formation of both the inner and the outer contractile

34 rings of the chloroplast. 


\section{Introduction:}

Chloroplasts are bound by outer and inner envelope membranes which enclose an additional compartment called the thylakoid. Specialized to capture the light energy available in sunlight and convert it into ATP and NADPH, thylakoids are responsible for the production of most of the oxygen in the Earth's atmosphere.

Structurally, thylakoids appear as an intricate network of columns of stacked discs, termed grana, interconnected by unstacked regions called stromal lamellae. This architectural complexity is further highlighted by the location of different proteins complexes within the different thylakoid regions. Photosystem II and the light-harvesting complex II trimer are found in the granal regions of the thylakoid, whereas the photosystem I - light harvesting complex I supercomplex and the ATP synthetase are found in the stromal lamellae; the cytochrome b6/f complex is located in both (Boardman et al., 1966; Boekema et al., 2000; van Roon et al., 2000; Daum et al., 2010). Spectroscopic studies , electron microscopy (Heslopharrison, 1963; Paolillo and Falk, 1966; Schoenknecht et al., 1990) and more recently, electron tomography (Shimoni et al., 2005; Daum et al., 2010; Austin and Staehelin, 2011) have shown that the thylakoid system within a single chloroplast consists of a single vesicle with its membrane intricately folded upon itself. A number of proteins, such as Thf1, VIPP1, FtsZ and FZL were shown to be essential for the development and maintenance of thylakoid structure (Kroll et al., 2001; Wang et al., 2004;

Gao et al., 2006; El-Kafafi et al., 2008; Karamoko et al., 2011; Lo and Theg, 2012). The mechanism that allows chloroplasts to sort and separate the intricate structures of the thylakoid membrane during organelle division remain unknown.

Chloroplasts division requires a multitude of proteins to assemble into rings along the center of the organelle and to constrict in synchrony (Maple-Grodem and Raynaud, 2014; 
61 Osteryoung and Pyke, 2014). The first ring that forms involves the stromal protein FtsZ

62 interacting with Arc6, a protein found in the inner envelope membrane (McAndrew et al., 2001;

63 Vitha et al., 2003). Arc6 has also been shown to interact with PDV2 in the outer envelope, which

64 along with PDV1, recruits DRP5 or Arc 5, a dynamin-related protein that forms a contractile ring

65 along the cytoplasmic side of the chloroplast (Yoshida et al., 2006; Glynn et al., 2008). FtsZ1

66 and isoforms of FtsZ2 in Arabidopsis have been found to associate with the thylakoid membrane

67 in a developmentally dependent manner (El-Kafafi et al., 2008; Karamoko et al., 2011). While

68 this is consistent with the involvement of these proteins with thylakoid division, this remains a

69 matter of speculation.

The separation of thylakoids during chloroplast division has been captured in electron

71 micrographs by a number of different groups (Leech et al., 1981; Oross and Possingham, 1989;

72 Robertson et al., 1996). Images show thylakoids dispersed throughout the chloroplast in the early

73 stages of plastid division. As division proceeds, the number of thylakoid grana and stromal

74 lamellae that extend across the length of the original plastid dwindles until a single segment

75 spans the isthmus of the constricted plastid. At the final stages of plastid division, the thylakoid

76 membrane along with one of the daughter organelles appear to be twisted so that the thylakoid

77 membrane loses its longitudinal orientation (Robertson et al., 1996). Thylakoid division appears

78 to precede the separation of the daughter organelles (Whatley, 1980). The mechanism of

79 thylakoid division, and its reliance on the chloroplast division cycle remain unknown. In this

80 study we dissect the dependence of thylakoid division on the chloroplast division machinery by

81 comparing the sizes of thylakoids found in chloroplast division mutants that are arrested at

82 different states of plastid division. 
84

85

86

87

\section{Results:}

\section{Experimental theory and design}

Wild type Arabidopsis plants possess 120 chloroplasts per mesophyll cell, each with a single thylakoid membrane (Pyke and Leech, 1994). The composition of thylakoids from chloroplast mutants that are unable to divide is unknown. We first examined the effect of the lack of an inner contractile ring on the division of thylakoids. The chloroplast division mutant, $\operatorname{arc} 6$, has a premature stop codon near the amino-terminal region of the protein, thereby preventing the formation of an inner contractile ring and rendering the plastid incapable of division (Vitha et al., 2003). As a result, the mutant plant possess giant-sized chloroplasts (Pyke et al., 1994). We reasoned that there are two possible scenarios that can describe the fate of the thylakoids in $\operatorname{arc} 6$ mutants. In the first, we assume that the thylakoids in the $\operatorname{arc} 6$ chloroplasts are still capable of undergoing division independent of the chloroplast division cycle. As a result, the number and size of the thylakoids within the giant $\operatorname{arc} 6$ chloroplasts will likely be similar to those found in wild-type cells. In the second scenario, we assume that the $\operatorname{arc} 6$ thylakoids cannot divide independently of the chloroplast division cycle. Accordingly, the arc6 chloroplasts would possess a single giant-sized thylakoid.

We sought to determine the relative sizes of the thylakoids in $\operatorname{arc} 6$ and wild type chloroplasts by measuring the sensitivity of the ionic conductivity of the thylakoid membrane to the pore-forming ionophore gramicidin. This experiment, originally performed by (Schoenknecht et al., 1990), is based on the ability of a small number of gramicidin pores to short circuit the capacitance of a thylakoid membrane vesicle. Titration of the membrane capacitance with gramicidin will report on the size of the thylakoid electrical unit, with larger thylakoids being more readily short-circuited by a given concentration of ionophore than smaller 
thylakoids. The conductivity of the thylakoid membrane can be conveniently and non-invasively monitored via the well-characterized decay of the carotenoid electrochromic shift at $520 \mathrm{~nm}$ induced by a short pulse of light (Junge and Witt, 1968; Witt, 1979; Bailleul et al., 2011). By this technique it was determined that the size of the thylakoid electrical unit within chloroplasts corresponds to all the photosynthetically active membranes within each plastid; that is, there is essentially one thylakoid per chloroplast (Schoenknecht et al. 1990). This conclusion has been confirmed by TEM micrographs of serially sectioned plastids (Heslopharrison, 1963; Paolillo and Falk, 1966; Mustardy and Janossy, 1979), and more recently, by electron tomography (Nierzwicki-Bauer et al., 1983; Shimoni et al., 2005; Mustardy et al., 2008; Austin and Staehelin, 2011; Daum and Kuhlbrandt, 2011).

\section{The electrical unit in arc6 chloroplasts is larger than that in wild-type chloroplasts}

It would be expected that large thylakoid vesicles potentially formed in giant chloroplasts might be susceptible to disruption by shear forces applied during isolation. In order to minimize this possibility, protoplasts were made from both wild type and arc6 plants and chloroplasts were subsequently isolated therefrom. Figure 1 shows that despite the expectation that the giant single chloroplasts present in the arc6 mutants would be even more fragile than those in the wild type, they could be isolated intact from protoplasts by incubation in a carbonate-containing buffer. This is, to our knowledge, the first successful isolation of these giant chloroplasts with their envelopes intact.

We then probed the size of the thylakoid electrical units via the light-induced carotenoid electrochromic signal at $520 \mathrm{~nm}$. After a $9 \mathrm{~ms}$ light pulse, the $\Delta \psi$-indicating $\Delta \mathrm{A}_{520} \mathrm{~nm}$ signal exhibited biphasic relaxation kinetics in both wild type and arc6 plastids. Arc6 chloroplasts 
131 displayed a faster $\Delta \psi$ relaxation rate than the wild type even in the absence of ionophore (Figure

132 2A-B). The addition of gramicidin to the chloroplasts resulted in an accelerated relaxation rate

133 of the $\Delta \mathrm{A}_{520} \mathrm{~nm}$ signal in both wild type and arc6 samples (Figure 2A-B), with increasing

134 concentrations resulting in faster decays. This was also conveniently manifested in the initial

135 point recorded as the $9 \mathrm{~ms}$ actinic pulse was turned off. Since a $9 \mathrm{~ms}$ pulse is not a single

136 turnover flash, the magnitude of the electric field measured at $9 \mathrm{~ms}$ results from competition

137 between field generation by multiple reaction center excitations and decay by ion counter

138 movement, the latter of which is accelerated by gramicidin. Accordingly, thylakoid membranes

139 with increased ion permeability display a lower magnitude of the $\Delta \mathrm{A}_{520} \mathrm{~nm}$ signal at $9 \mathrm{~ms}$.

140 Acceleration of the electrochromic shift decay can be clearly seen by plotting the magnitude of

141 the $9 \mathrm{~ms} \Delta \mathrm{A}_{520} \mathrm{~nm}$ absorbance as a function of the logarithm of the gramicidin concentration

142 (Figure 2C). This plot reveals the increased sensitivity of the thylakoid conductance to

143 gramicidin exhibited by arc6 chloroplasts over those from wild-type plants, and indicates that the

144 thylakoids are larger in the arc6 mutant.

\section{Arc6 chloroplasts possess giant thylakoids}

When isolated protoplasts are placed in very low osmotic conditions, the plasma

148 membrane and the chloroplast outer and inner envelope membranes break, resulting in the

149 release of the thylakoids (Mercer, 1954; Weier et al., 1965; Hinnah and Wagner, 1998).

150 However, thylakoid membranes are more resilient to osmotic pressure than the rest of the cell's

151 membranes, and thylakoids swell to form blebs. We used this property of thylakoids to provide

152 an independent measurement of the size of wild type and arc6 thylakoids. In the confocal

153 microscope, chlorophyll autofluorescence serves as a marker for thylakoid membranes (Figure 
3). Figure 3B shows a representative cross section image of a bleb formed from arc6 thylakoids, and it is apparent that it is significantly larger than those formed from wild-type thylakoids (Figure 3A). The diameter of the wild type bleb (Figure 3A) is $\sim 12.8 \pm 3.9 \mu \mathrm{m}$, whereas the arc6 bleb (Figure 3B) diameter averages $21.7 \pm 11.2 \mu \mathrm{m}$. While the diameter of a spherical object such as a bleb observed in a single cross section could be mistakenly underestimated by examining an image off of the equatorial plane, those images in Fig. 3 were produced after scanning back and forth through the z-plane to find the maximum observed diameters. Representative z-projections, in which all z-plane images are stacked one upon each other, confirm the dramatic difference in sizes between $\operatorname{arc} 6$ and wild type blebs (Figure 4A-B). The values in Table 4.1 report the average diameters of 1000 bleb samples. There was, however, a remarkable variation in the sizes of the blebs formed from these (and other) plants, and this is seen clearly in the diameter distribution histograms in Figure 5A. The possible origins of this variation are examined in the Discussion.

Together with the measurements of the electrical unit size above, the confocal microscopy images in Figures 3 and 4 show that arc6 chloroplasts possess giant thylakoids. This suggests that thylakoid division is dependent on chloroplast division.

\section{Incomplete formation of contractile rings result in giant thylakoids}

To test the dependence of thylakoid division on the constriction of the outer contractile ring, we examined two chloroplast division mutants, $p d v 1$ and $p d v 2$, which exhibit 2-6 gigantic chloroplasts that possess a dumbbell-like structure. These proteins reside in the outer envelope membrane and act as a functional pair to interact with Arc 5 and form the outer contractile ring. The $p d v$ mutant chloroplasts are still able to form the inner contractile ring, however without the outer ring the plastids never divide (Miyagishima et al., 2006). We tested two alleles of each $p d v$ 
178

179

180

181

182

183

184

185

186

187

mutant, one that contained a mutation close to the $\mathrm{N}$-terminus and another to the $\mathrm{C}$ - terminus which we labeled -1 and -2 , respectively. On average, $p d v 1-1, p d v 1-2, p d v 2-1$, and $p d v 2-2$ blebs (Figure 3C-F) were much larger than wild-type blebs. The blebs found in these mutants were slightly smaller than those found in the $\operatorname{arc} 6$ mutant $(\sim 20 \mu \mathrm{m}$ vs $\sim 22 \mu \mathrm{m}$, Table 1$)$.

Representative z-projections show the $p d v$ blebs in their entirety and provide further evidence that the blebs are much larger than those found in wild type (Figure 4C-F). The variations observed in bleb sizes for the wild type and arc6 plants were evident in these mutants as well (Figure 5B).

To examine the behavior of thylakoids in a mutant that undergoes asymmetric plastid division we measured bleb sizes from an $\operatorname{arc} 3$ mutant (Figure $3 \mathrm{G}$ ) in which the inner contractile ring is misplaced (Pyke and Leech, 1994; Zhang et al., 2013). The arc3 mutant plastids possess 15 chloroplasts per cell (Burch-Smith et al., 2007). Here we expected the bleb sizes to average closer to those of the wild-type blebs, but with a larger variation, as an asymmetric division should result in one larger-than-wild-type and one smaller-than-wild-type chloroplast. As per these expectations, the arc3 bleb sizes averaged $15.6 \mu \mathrm{m}$, somewhat larger than the wild-type blebs, and displayed a standard deviation of $\pm 6.5 \mu \mathrm{m}$ (Table 1, Figure 5C). While we obtained a similar large standard deviation of bleb sizes from the giant chloroplast mutants, we note that blebs formed from the arc3 mutants are only $22 \%$ larger than those from wild type plants, but display a $67 \%$ increase in the diameter standard deviation.

\section{These results in aggregate reveal that thylakoids do not possess an independent division} machinery and cannot divide if the chloroplasts cannot divide. 


\section{Discussion:}

202 Thylakoid division is dependent on the chloroplast division machinery

Thylakoids represent a membrane system essential to shaping and maintaining the

biosphere. Yet, the reproduction and partitioning of thylakoids, which must occur multiple times in green plants during each cell cycle, has so far escaped much scrutiny. In this work we have examined the question of whether thylakoids possess their own division machinery that operates independently of the chloroplast reproduction events.

219 (Schoenknecht et al., 1990). At any given ionophore concentration, it is expected that a larger vesicle would incorporate more gramicidin pores per vesicle than would a smaller one.

221 Experimentally, this would be manifested as an increased sensitivity of the decay of the

222 membrane potential to gramicidin. Using the amplitude of the carotenoid electrochromic signal 223 at the end of a $9 \mathrm{~ms}$ illumination pulse as an indicator of the membrane conductivity, we found 
224 that the arc6 thylakoids were indeed considerably more sensitive to gramicidin addition in the

225 manner suggested. From this we conclude that thylakoid vesicles in the arc6 mutant are larger

226 than those in the wild type. This would imply that the thylakoids cannot divide independently of

227 plastid division.

It should also be noted that the ratio of gramicidin/chlorophyll required to accelerate the

229 rate of electric field dissipation is higher in our studies than what was previously reported by

230 (Schoenknecht et al., 1990). This discrepancy can be accounted for as a result of differences in

231 thylakoid preparations. The thylakoids utilized in our studies were prepared by lysing intact

232 chloroplasts within the same chamber that was carried out for taking the $\Delta \mathrm{A}_{520} \mathrm{~nm}$

233 measurements. As a result, the thylakoid samples contained additional chloroplast envelope

234 membranes which absorbed gramicidin and increased the working gramicidin/chlorophyll ratio

235 up to 50 fold (Nishio and Whitmarsh, 1991). Thus the higher gramicidin concentrations used in

236 this study are to be expected. Additionally, differences in gramicidin's dimerization constants in

237 thylakoids from different plant species can also contribute to this discrepancy (Schoenknecht et

238 al., 1992). The difference in membrane permeability between the arc6 and wild type thylakoids

239 exhibited in Figure 2A-B may result from arc6 thylakoids possessing naturally more ionically

240 conductive membranes. It is also likely that the chloroplast solution used for the ECS

241 measurements contained intact and broken chloroplasts which may have resulted in a slightly

242 accelerated $\Delta \mathrm{A}_{520} \mathrm{~nm}$ decay due to the damage that the thylakoids may have sustained during the

243 event that caused the lysis of the intact chloroplast. However, the sensitivity of the thylakoids

244 towards increasing concentrations of gramicidin would not be expected to be due to the presence

245 of lysed chloroplasts. Accordingly, our observation that the arc6 thylakoids are more sensitive 
towards increasing amounts of gramicidin indicates that the $\operatorname{arc} 6$ thylakoids are larger than their wild type counter parts.

The sizes of osmotically lysed thylakoids from the different chloroplast division mutants provided an independent measurement of thylakoid structure. It is apparent that the thylakoids formed from arc6 chloroplasts were considerably larger than wild-type blebs. The average diameter of the wild-type bleb was $12.8 \mu \mathrm{m}$, whereas that of $\operatorname{arc} 6$ blebs was $21.7 \mu \mathrm{m}$ (Table 1). Our similar findings with the $p d v 1$ and $p d v 2$ mutants suggests that thylakoid division is closely tied to the chloroplast division process (Table 1). The arc6 chloroplasts are unable to form contractile rings, whereas the $p d v 1$ and $p d v 2$ mutants are capable of forming an inner contractile ring but only a partial outer ring. Our results suggest that separation of thylakoid membranes depends on the constriction forces applied by the chloroplast division machinery. The sizes of the $\operatorname{arc} 3$ blebs show that thylakoid division is closely tied with the final stages of plastid division since the aberrant placement of the plastid ring leads to a rather large spread in thylakoid bleb sizes. Thus, our results from the bleb studies agree with the previously published electron microscopy images showing that thylakoid division occurs at the late stages of plastid division (Whatley, 1980; Robertson et al., 1996).

It is noteworthy that we observe a distribution of bleb sizes in our experiments (Figure 5), even in the wild type plants, the reasons for which may be manifold. First, the plastid division mutants we examined do not display giant chloroplasts in every cell type. Measurements of chloroplasts size found in the arc6 guard cells made by (Pyke et al., 1994) and (Robertson et al., 1995) show that the arc6 chloroplasts in the guard cells are smaller than the ones observed in mesophyll cells. Similarly, guard cells in $p d v$ mutants also have smaller plastids compared to mesophyll cells (our observation). All cell types would have been in our preparations. Upon 
269

270

271

272

273

274

275

276

277

278

279

280

281

282

283

284

285

286

287

288

289

290

291

reflection one sees that a small number of cells with a normal allotment of chloroplasts mixed in with a preponderance of cells containing one and or a few giant chloroplasts would lead to a skewed bleb size distribution, as seen in our experiments. Second, it is clear from the patchiness of the observed chlorophyll fluorescence that thylakoids do not unstack and unravel completely during bleb formation, and incompletely expanded thylakoids would form smaller blebs. This incomplete unfolding was not a consequence of short incubations in water, as neither did the observed patchiness decrease (not shown), nor did the bleb diameters increase with longer incubation times (Figure S1). Third, while we made every effort to be gentle with the samples, we cannot be sure that we did not cause some breakage of the blebs during handling. We would expect osmotically swollen vesicles to be more fragile than normal thylakoids, and any such breakage would necessarily result in the formation of smaller blebs. Fourth, budding as an alternative plastid division mechanism has been observed in the tomato suffulta mutant (Chen et al., 2009), and Bryophyllum pinatum (Kulandaivelu and Gnanam, 1985). In a study that targeted GFP to the stroma of arc6 mutant chloroplasts, small vesicular bodies that contained GFP were suggested to have budded off of the giant chloroplasts (Forth and Pyke, 2006). Such buds would be expected to contain smaller thylakoid vesicles. Finally, there is inherent variability in our samples. Wild type Arabidopsis cells do not always contain 100 chloroplasts, arc6 mutant cells do not always contain one chloroplast, and $p d v 1$ mutant cells do not always contain three chloroplasts. Instead, these are averages. Thus we would expect some variability in bleb sizes even if the other factors mentioned above were not in play. We hold that the fact that we see any giant blebs at all in giant chloroplast mutants provides strong evidence that thylakoid division and chloroplast replication are not independent and uncoupled events. 


\section{Thylakoid division mechanism}

We propose that the constriction of the envelope membrane by the chloroplast division machinery acts to partition portions of the thylakoid into the two different poles of the dividing organelle. To reach this stage wherein a small portion of the thylakoid vesicle is trapped in the central isthmus of the dividing chloroplast, proteins that are known to be involved in thylakoid

297 formation and remodeling, such as Vipp1, Thf1, FtsZ and FZL (Kroll et al., 2001; Wang et al., 2004; Gao et al., 2006; El-Kafafi et al., 2008; Lo and Theg, 2012), might be brought to bear on

the thylakoid structure. These proteins have been proposed to govern the fission and fusion events accompanying stacking and unstacking of the thylakoid membrane in response to different light conditions (Chuartzman et al., 2008), and are thus candidates for those that might be involved in the final separation of the thylakoid vesicle into the two daughter plastids. Mesophyll cells in which the thylakoid protein FtsZ1, FtsZ2-2 and FZL has been knocked out possess fewer and larger chloroplasts compared to wild type cells (Gao et al., 2006; El-Kafafi et

311 responsible for dividing the thylakoid membrane as well. It will surely be interesting to elucidate

312 through future studies the mechanism through which these forces generated on one membrane 313 system are transduced to the other. 


\section{Materials and Methods:}

316 Plant material, protoplast and chloroplast isolation

317 The Col-0 ecotype of Arabidopsis thaliana was used as the wild type. The chloroplast division mutants, $\operatorname{arc6}$ (SAIL_693_G04), $p d v 1-1, p d v 1-2, p d v 2-1(\mathrm{SALK} 059656)$, and $p d v 2-2$ seeds were obtained from ABRC. All plants were genotyped and only homozygous lines were used (see supplemental materials for further detail). All plants were grown for 5 weeks on

322 Murashige and Skoog (Phytotechnologies, Santa Cruz, CA) agar before harvesting the tissue for 323 chloroplasts (Theg and Tom, 2011); growth chamber conditions were $20^{\circ} \mathrm{C}$ with 16 hrs light cycle of $100 \mu \mathrm{mol}$ photons $/ \mathrm{m}^{2} / \mathrm{sec}$ at $60 \%$ humidity. Plant tissue was harvested following the procedure described previously (Fitzpatrick and Keegstra, 2001) with some minor modifications. Briefly, plants were minced in a petri dish and washed with digestion buffer (400 mM sorbitol, g/mL cellulase 'onozuka' R-10 and 0.01 g/mL macerozyme R-10 (Yakult Pharmaceutical Ind, Tokyo, Japan) for $3 \mathrm{hrs}$ at room temperature with gentle rocking. Digested samples were passed

330 through 4 layers of cheese cloth, and then centrifuged at $370 \times g$ for 5 minutes at $4^{\circ} \mathrm{C}$. The pellet was gently resuspended in resuspension buffer $(400 \mathrm{mM}$ sorbitol, $0.5 \mathrm{mM} \mathrm{CaCl}, 20 \mathrm{mM} \mathrm{Mes-}$ $\mathrm{KOH}, \mathrm{pH}$ 6.0). Samples were centrifuged again at $660 \mathrm{x} g$ for 5 minutes at $4^{\circ} \mathrm{C}$, and if blebs were

334 ice in the dark until further use. 
through a syringe 4 times. For arc6 chloroplast isolation, the protoplast pellet from the arc6 tissues was resuspended in breakage buffer and incubated for 5 minutes on ice in the dark. Both samples were then passed through a Percoll gradient containing half volume of Percoll and half volume of grinding buffer (330 mM Sorbitol, $1 \mathrm{mM} \mathrm{MgCl}, 1 \mathrm{mM} \mathrm{MnCl} 2,2 \mathrm{mM}$ EDTA, $0.1 \%$ BSA, $50 \mathrm{mM}$ Hepes- $\mathrm{KOH}, \mathrm{pH} 7.3$ ) at $8035 \mathrm{x} \mathrm{g}$ at $4^{\circ} \mathrm{C}$. Intact chloroplasts were removed from the bottom band, and washed with a storage buffer (330 mM Sorbitol, $50 \mathrm{mM}$ Hepes-KOH, $\mathrm{pH}$ 8). Chloroplasts were centrifuged at $1475 \mathrm{x} g$ at $4^{\circ} \mathrm{C}$ for $5 \mathrm{~min}$. This step was repeated again to remove the remaining Percoll. After chlorophyll determination (Arnon, 1949), isolated chloroplasts were stored in the storage buffer on ice in the dark until used.

\section{Electrochromic shift measurements}

All electrochromic shift measurements contained $0.02 \mu \mathrm{g} / \mu \mathrm{L}$ chlorophyll concentration, $1 \mathrm{mM}$ methyl viologen, and various amounts of gramicidin D (Sigma-Aldrich, St. Louis, Missouri); measurements were performed in $1.2 \mathrm{~mL}$ of storage buffer. Gramicidin stock solutions were prepared in ethanol; the total percentage of ethanol did not exceed $0.03 \%$ for all titrations. The measurements typically began with the addition of chloroplasts into a $2.5 \mathrm{ml}$ polystyrene cuvette (Fisher Scientific, Houston, Texas) containing a master mix of methyl viologen, storage buffer and gramicidin. Samples were mixed with a stir bar for 2 minutes at room temperature, and then transferred into a $1.5 \mathrm{~mL}$ Polystyrene cuvette (Fisher Scientific, Houston, Texas) with a $10 \mathrm{~mm}$ path length. The absorbance readings were performed using a JTS-10 LED pump-probe spectrometer (Bio-Logic SAS, Claix, France). All $520 \mathrm{~nm}$ absorption measurements consisted of a $1 \mathrm{sec}$ dark baseline followed by a $9 \mathrm{~ms}$ actinic pulse; the relaxation kinetics were followed out to 4 sec. 


\section{Thylakoid bleb formation}

363 Blebs were formed by diluting isolated protoplasts containing $2 \mu \mathrm{g}$ of chlorophyll into $2 \mathrm{~mL}$ of

364 doubly distilled water stored at $4^{\circ} \mathrm{C}$, and incubated on ice in the dark on ice for $1 \mathrm{hr}$ before

365 images were taken. Equal volumes of sample were loaded into a homemade perfusion chamber

366 consisting of $1.8 \mathrm{~mm} \times 100 \mathrm{~mm} \times 1.1 \mathrm{~mm}$ piece of polycarbonate and a $50 \times 22 \mathrm{~mm}$ coverslip

367 (Fisher Scientific Houston, TX) which had been coated with poly-L-lysine (Sigma-Aldrich St.

368 Louis, MO). Samples were centrifuged for 15 minutes at 60 x $g$ using a GS-6KR swinging

369 bucket centrifuge (Beckman Coulter Inc., Brea, CA) at $10^{\circ} \mathrm{C}$ and imaged.

\section{Microscopy}

372 Phase contrast microscopy was performed with a Zeiss Standard 25 ICS microscope. Images at

373 160x magnification were taken by placing a camera, Canon powershot A620 (Canon, Melville,

374 New York), into the ocular eyepiece. Fluorescence microscopy was performed using a Zeiss

375 LSM 710 confocal microscope (Zeiss, Oberkochen, Germany). Image compilation and analysis

376 were performed using Fiji software. Bleb diameters were measured from populations of isolated

377 blebs taken from protoplast isolation preparations. The reported bleb diameter size were

378 determined by scanning through the z-stack and finding the maximal diameter of the bleb. All

379 reported values for bleb diameters were determined as being the largest distance from one end of

380 the membrane to the other throughout the entire $\mathrm{z}$-axis per bleb.

381

\section{Supplemental Material}


384 Figure S1. Thylakoid blebbing time course

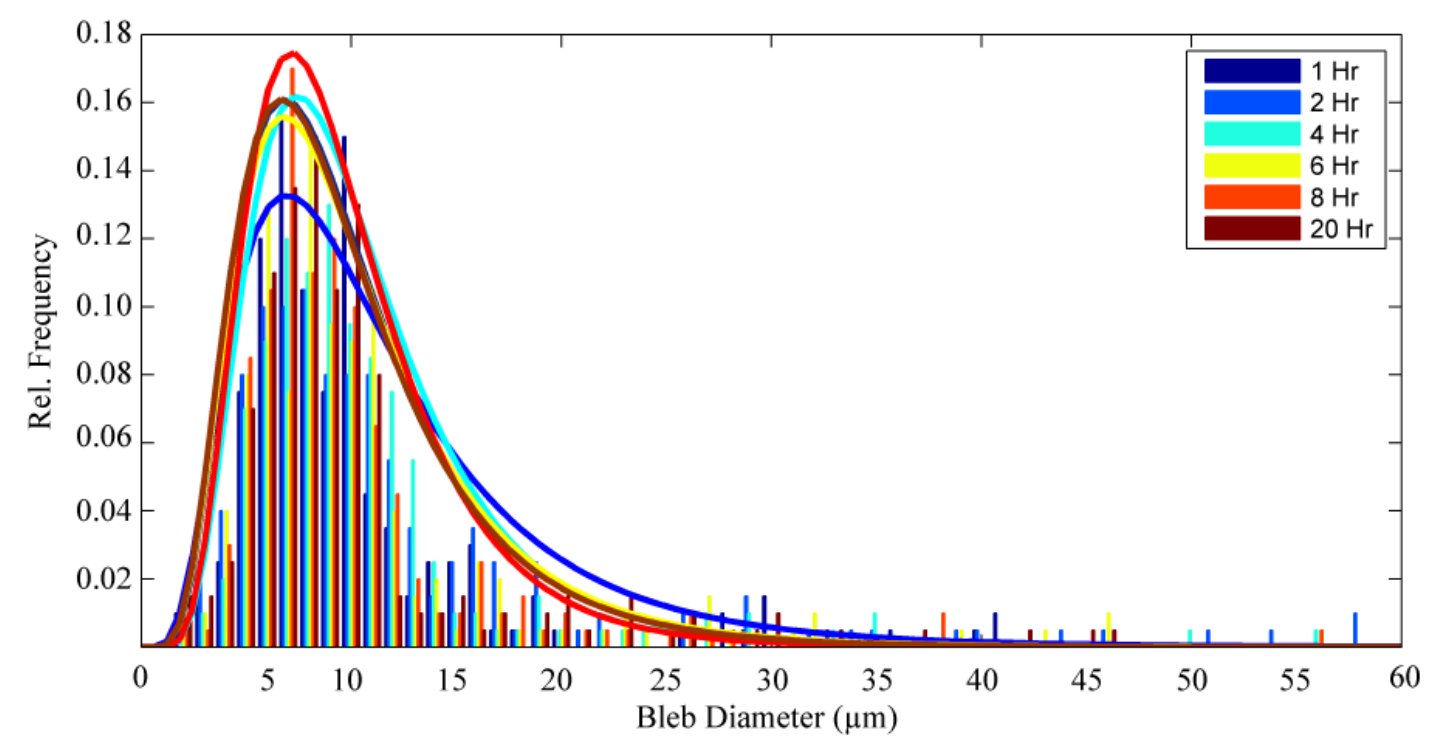

Figure S1. Thylakoid blebbing time course

387 A histogram reporting the frequency of bleb diameter sizes from a mixture of arc6 and wild type samples over the course of 20 hours. ( $\mathrm{n}=200$ for all time points) The plots were fit with a lognormal distribution function. 
391 Table S1. Table displaying the primers utilized to genotype plants from the T-DNA insertion

392 lines, arc6, and pdv2 -1, pdv2-2.

393 Plants from the T-DNA insertion lines, arc6, and $p d v 2-1, p d v 2-2$, were genotyped using the 394 following primers:

\begin{tabular}{|l|l|}
\hline Genotype & $5^{\prime}-3^{\prime}$ Primer Sequence \\
\hline arc6 & FP: ATCAGCAACGGACATTTCAAC \\
\hline arc6 & RP: TAAATGGTTTAAGCGGTGTGC \\
\hline$a r c 6$ & SAIL LB1: GCCTTTTCAGAAATGGATAAATAGCCTTGCTTCC \\
\hline$p d v 2-1$ & FP: AATTACTTGTCTCTCTTCTCTTCCCCACCA \\
\hline$p d v 2-1$ & SALK LB1.3: ATTTTGCCGATTTCGGAAC \\
\hline$p d v 2-1$ & FAIL LB1: GCCTTTTCAGAAATGGATAAATAGCCTTGCTTCC \\
\hline$p d v 2-2$ & RP: ATACTACTCAAATGAATCTCTCCCTCATCCA \\
\hline$p d v 2-2$ & \\
\hline$p d v 2-2$ & \\
\hline
\end{tabular}


397 Table S2. Table displaying the primers utilized to genotype $P d v 1-1, p d v 1-I$, and $\operatorname{arc} 3$ plants by 398 detecting single nucleotide polymorphisms.

399

\begin{tabular}{|l|l|l|}
\hline Genotype & $5^{\prime}-3$ ' Primer Sequence & Digestion Enzyme \\
\hline$p d v 1-1$ & FP: AATCTCATCGCTAAGCTTGTCATGTAGAGC & SacI \\
\hline$p d v 1-1$ & RP: TTTTTTGTTGTGTCAATGAAATGGGAGAAA & SacI \\
\hline$p d v 1-2$ & FP: ACGTGTAGACAAGTTCGATCTCTGGTGTTAA & HpaI \\
\hline$p d v 1-2$ & RP: GCAGAAGAAAGAAGACAGATTCTACAGAAGCC & HpaI \\
\hline arc3 & FP: AACAAAACTTGTTCATTTGTCTTGCAGGGGTG & HphI \\
\hline arc3 & RP: GGCCTTCGCCACTGGCTTTTCCTTTCCAGA & HphI \\
\hline
\end{tabular}




\section{Acknowledgements:}

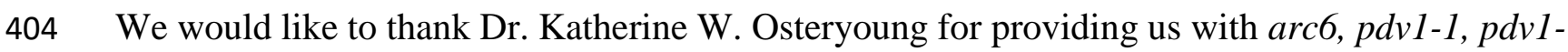

405 2, pdv2-1, and pdv2-2 Arabidopsis seeds, Drs. Bo Liu and Yuh-Ru Julie Lee for providing access

406 to their phase contrast microscope and for sharing their expertise in confocal microscopy. We

407 would also like to thank Drs. Lan-Xin Shi and Li Liu for their expertise in plant tissue culture.

408 This work was funded by the Division of Chemical Sciences, Geosciences, and Biosciences,

409 Office of Basic Energy Sciences of the US Department of Energy through Grant DE-FG02-

410 03ER15405.

411

412

413

414

415

416

417

418 
Table 1. Bleb Dimensions

420

421

\begin{tabular}{lc}
\hline Genotype & Diameter $(\boldsymbol{\mu m})$ \\
\hline wild type & $12.8 \pm 3.9$ \\
arc6 & $21.7 \pm 11.2$ \\
pdv1-1* & $19.5 \pm 8.6$ \\
pdv1-2* & $20.1 \pm 8.8$ \\
pdv2-1 & $18.9 \pm 6.9$ \\
pdv2-2* & $19.8 \pm 7.8$ \\
arc3 & $15.6 \pm 6.5$ \\
\hline & For all samples $n=1,000$
\end{tabular}

423 Table 1. Bleb Dimensions

424 A table reporting the average bleb diameters and standard deviations for all genotypes. All 425 values reported were statistically significant $(\mathrm{P}<0.05)$ from a student's t-test other than those

426 annotated with an (*). Samples annotated with $(*)$ are not statistically significant from each other $427 \quad(\mathrm{P}>0.05)$. 

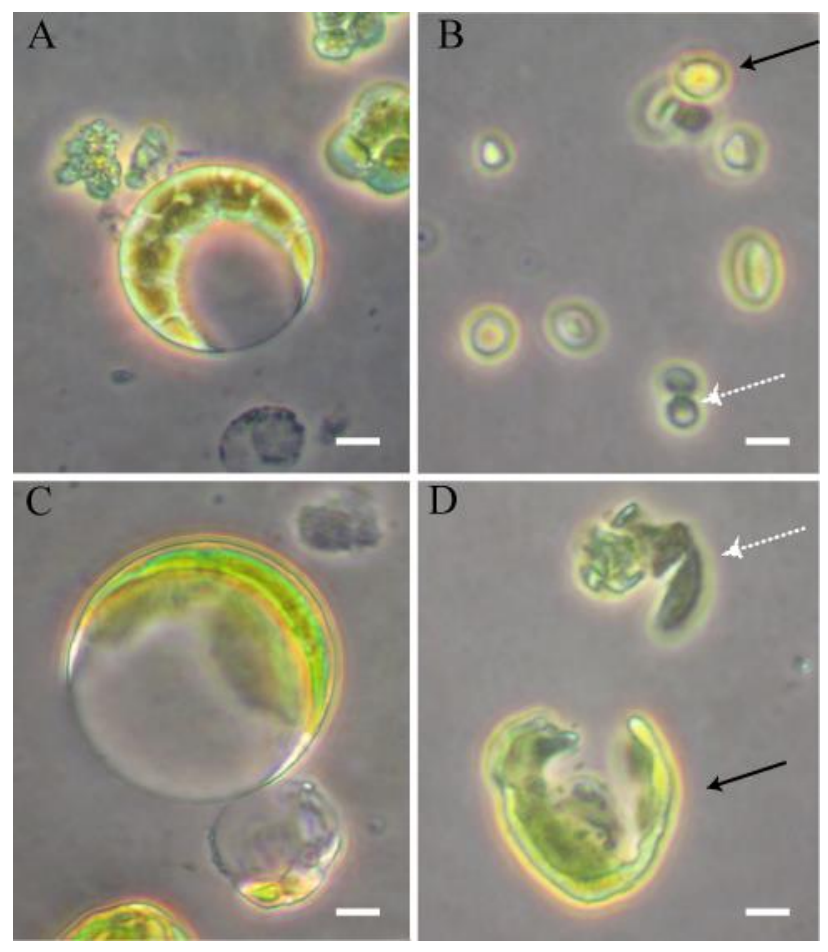

432 Figure 1. Isolation of intact chloroplasts.

433 A) and C), Isolated protoplasts from wild type and arc6 Arabidopsis under a phase contrast

434 microscope. B) and D), Wild type and arc6 isolated chloroplasts. Intact chloroplasts possess a

435 halo and are highlighted by the black arrow; broken chloroplasts are indicated by the white

436 arrow. Scale bar corresponds to $10 \mu \mathrm{m}$. 
A

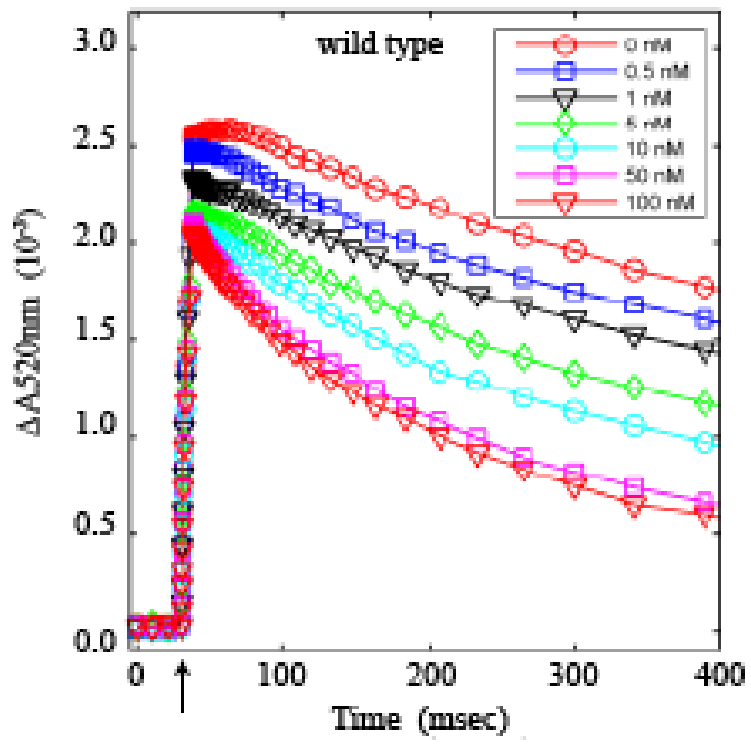

$\mathrm{C}$

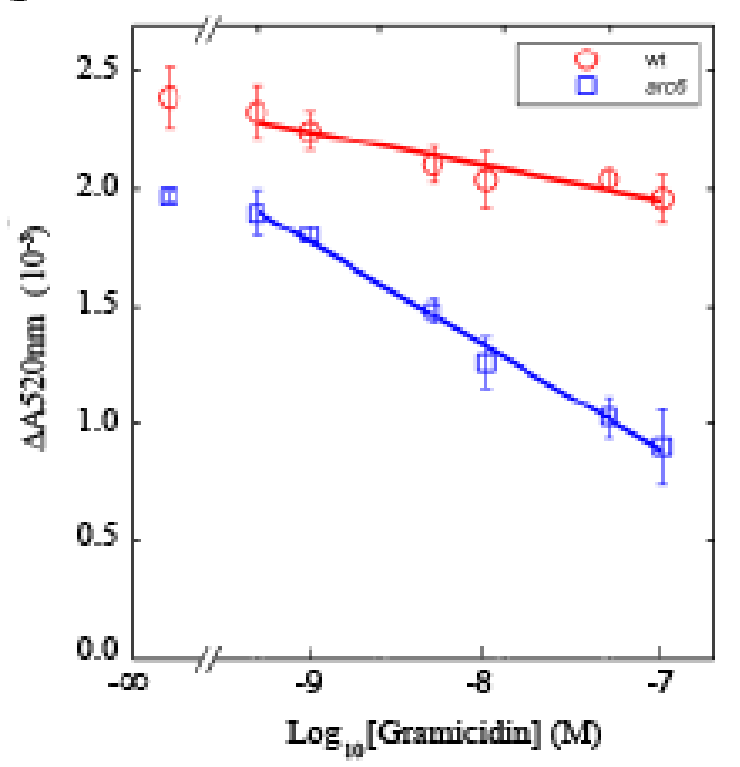

B

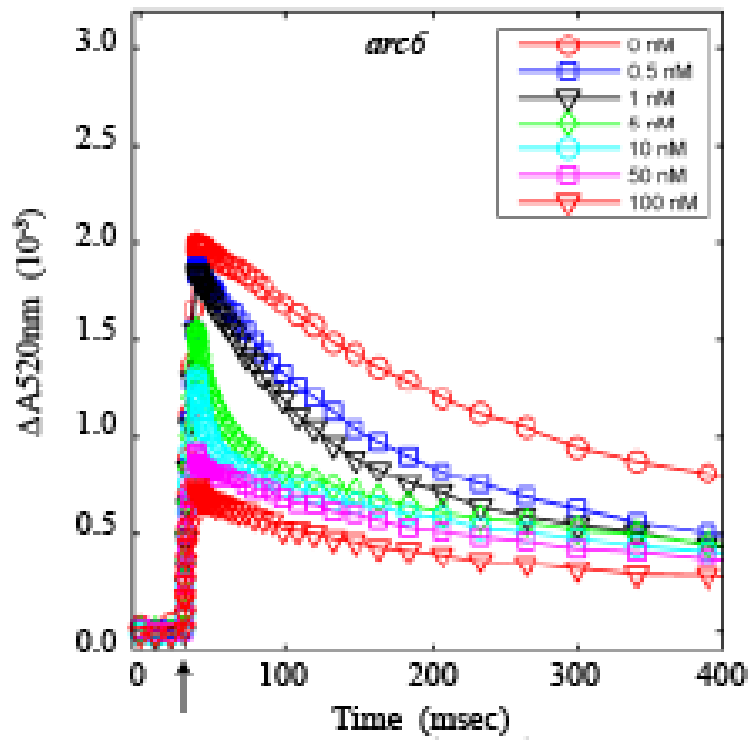

442 Figure 2. Thylakoid membrane conductivity measured by the electrochromic shift of

443 carotenoids.

444 Representative $\Delta \mathrm{A}_{520} \mathrm{~nm}$ measurements of A) wild-type and B) $\operatorname{arc6}$ chloroplasts with 445 increasing amounts of gramicidin; the arrow indicates the initiation of the $9 \mathrm{~ms}$ actinic flash. C) 
446 Plot of the first point of the $\Delta \mathrm{A}_{520} \mathrm{~nm}$ signal after the $9 \mathrm{~ms}$ actinic flash vs. the log of gramicidin

447 concentration. Bars indicate standard deviation of $n=9$ for each data point. The wild-type (red

448 circles) and $\operatorname{arc} 6$ (blue squares) data points fit by linear regression; the $\Delta \mathrm{A}_{520} \mathrm{~nm}$ values for $0 \mathrm{nM}$

449 gramicidin values are plotted to the left of the axis break and not utilized in the fit.

450

451 


\section{$452 \quad$ Ho et al. Figure 3}
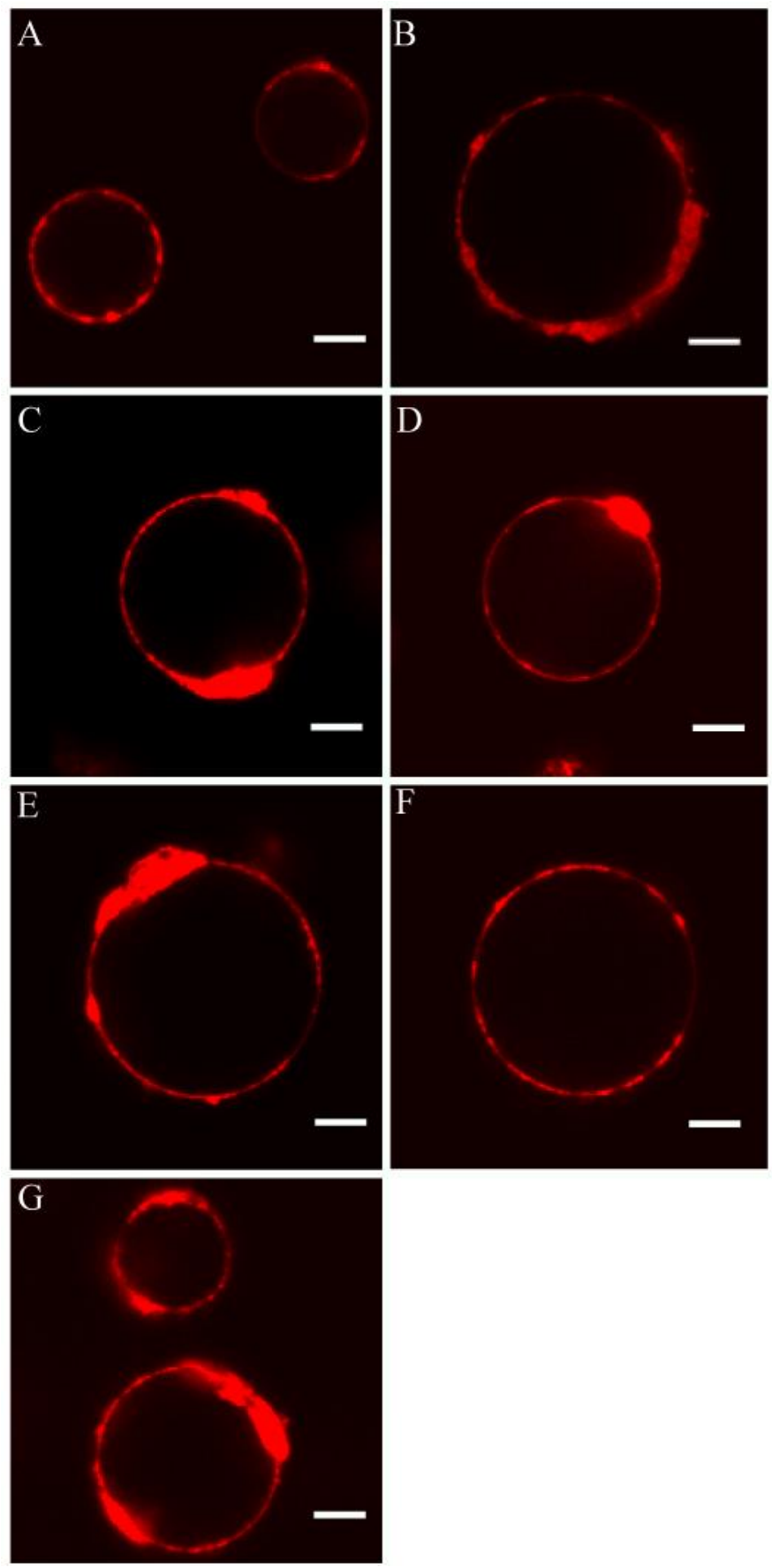

455 Figure 3. Chloroplast division mutants contain thylakoids that are much larger than those in

456 wild-type plants. 
457 A-G) Representative cross-sections of osmotically swollen thylakoids from wild-type, arc6,

$458 \quad p d v 1-1, p d v 1-2, p d v 2-1, p d v 2-2$, and arc3 plants, respectively, were taken using a laser scanning

459 confocal microscope. Chlorophyll autofluorescence is used to track the presence of the thylakoid

460 membrane. The scale bar corresponds to $10 \mu \mathrm{m}$.

461

462 


\section{Ho et al. Figure 4}
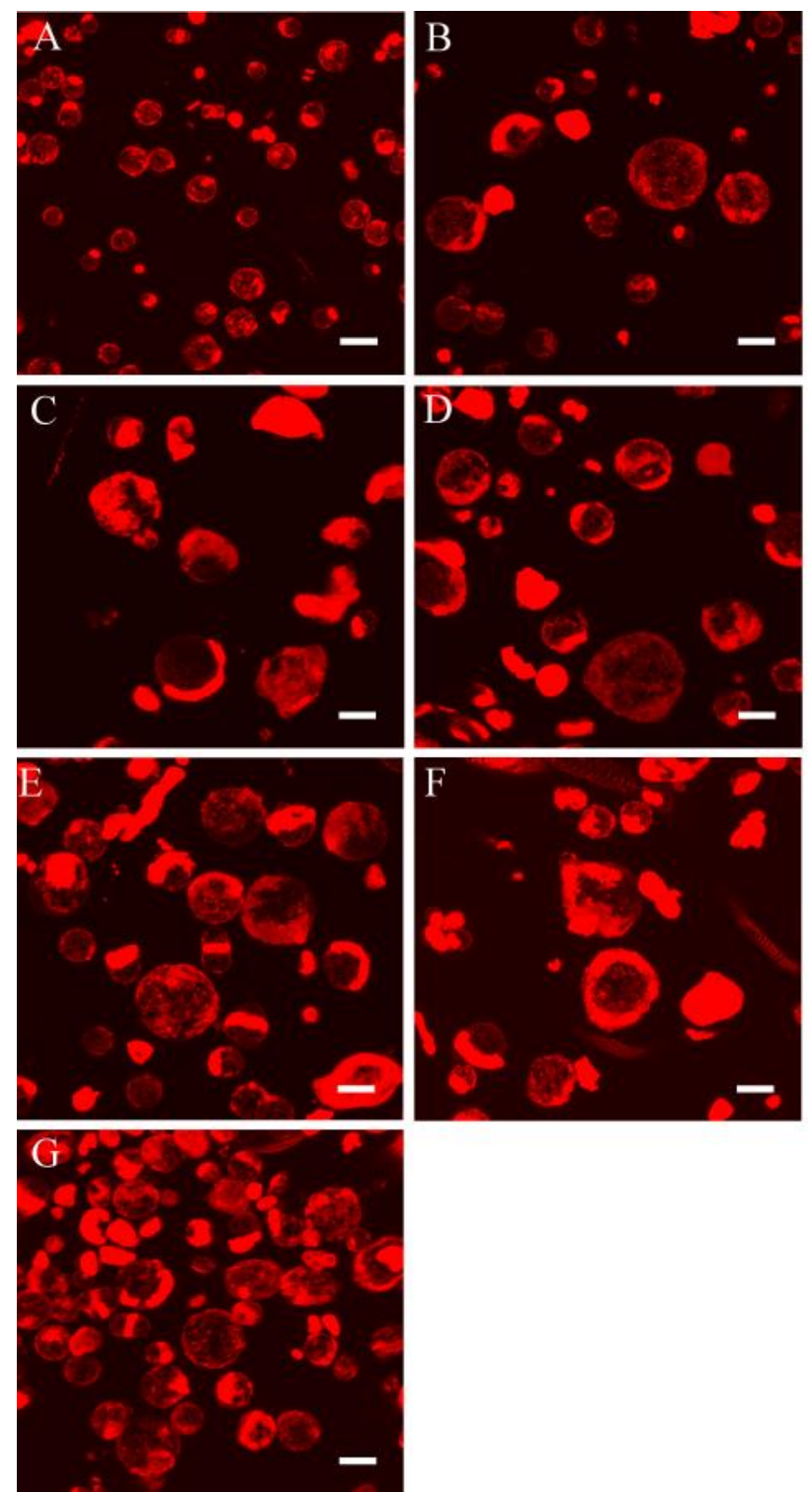

Figure 4. Z-projections of various thylakoid division mutants

467 Representative z- projections of osmotically swollen thylakoids from A) wild-type, B) $\operatorname{arc6}, \mathrm{C}$ )

$468 p d v 1-1$, D) $p d v 1-2$, E) $p d v 2-1$, F) $p d v 2-2$, and G) $\operatorname{arc} 3$ plants were taken using a laser scanning 469 confocal microscope. The scale bar corresponds to $20 \mu \mathrm{m}$. 


\section{$471 \quad$ Ho et al. Figure 5}

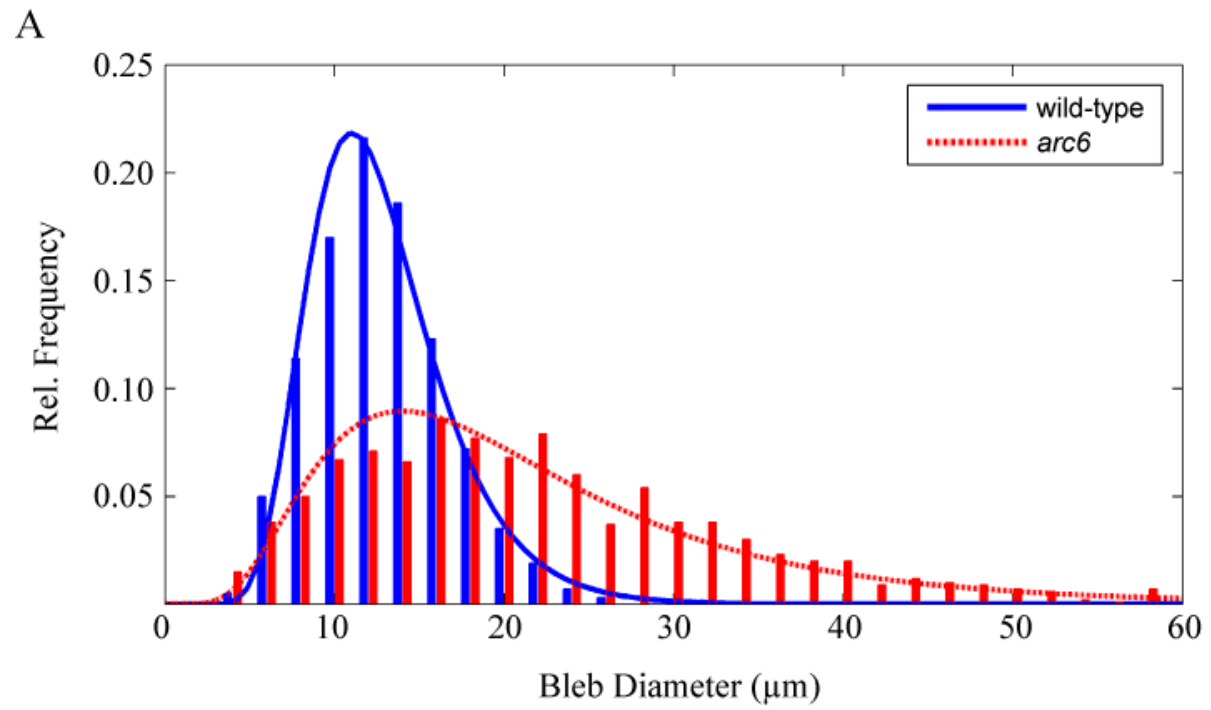

B

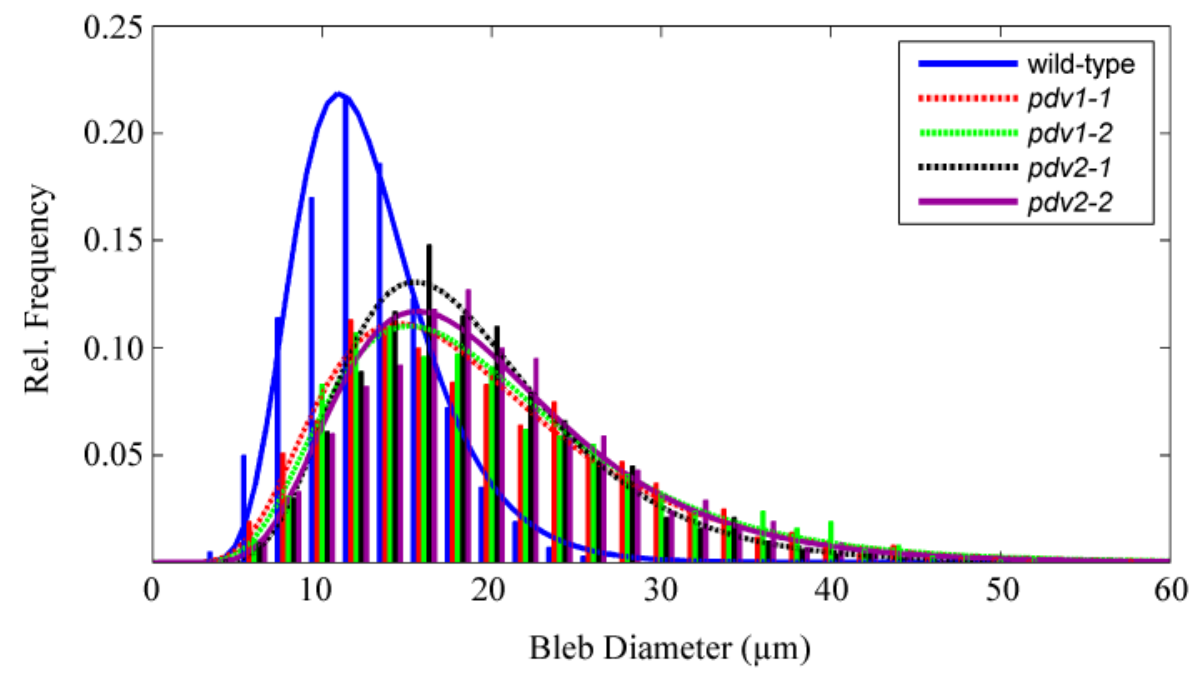

$\mathrm{C}$

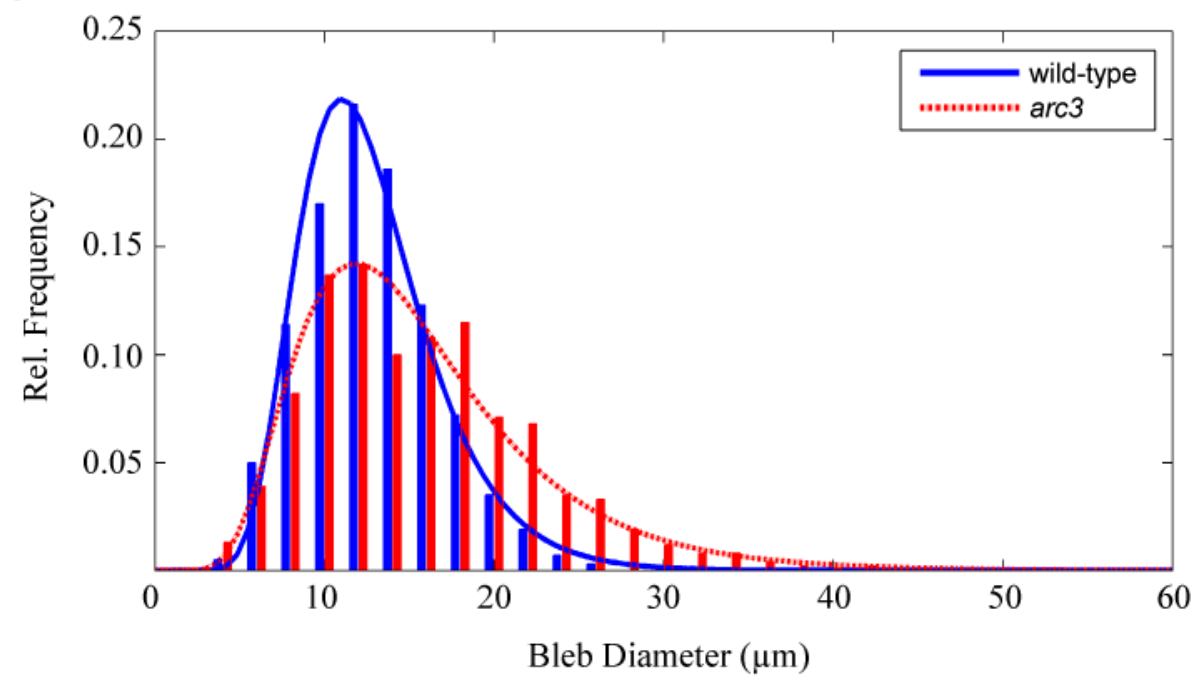


474 Figure 5. Thylakoid bleb population size distribution histogram

475 A) A histogram comparing the frequency of bleb diameter sizes between the wild type and A)

$476 \operatorname{arc} 6, \mathrm{~B})$ all $p d v$ mutants, and C) $\operatorname{arc} 3$ plants ( $=1,000$ for all genotypes). The plots were fit with

477 a log-normal distribution function.

478

479 


\section{References:}

Arnon DI (1949) Copper Enzymes in Isolated Chloroplasts. Polyphenoloxidase in Beta Vulgaris. Plant Physiol 24: 1-15

Austin JR, 2nd, Staehelin LA (2011) Three-dimensional architecture of grana and stroma thylakoids of higher plants as determined by electron tomography. Plant Physiol 155: 1601-1611

Bailleul B, Cardol P, Breyton C, Finazzi G (2011) Electrochromism: a useful probe to study algal photosynthesis (vol 106, pg 179, 2010). Photosynthesis Research 110: 151-152

Boardman NK, Thorne SW, Anderson JM (1966) Fluorescence Properties of Particles Obtained by Digitonin Fragmentation of Spinach Chloroplasts. Proceedings of the National Academy of Sciences of the United States of America 56: 586-\&

Boekema EJ, van Breemen JFL, van Roon H, Dekker JP (2000) Arrangement of photosystem II supercomplexes in crystalline macrodomains within the thylakoid membrane of green plant chloroplasts. Journal of Molecular Biology 301: 1123-1133

Burch-Smith TM, Schiff M, Caplan JL, Tsao J, Czymmek K, Dinesh-Kumar SP (2007) A novel role for the TIR domain in association with pathogen-derived elicitors. Plos Biology 5: 501-514

Chen YL, Asano T, Fujiwara MT, Yoshida S, Machida Y, Yoshioka Y (2009) Plant Cells Without Detectable Plastids are Generated in the crumpled leaf Mutant of Arabidopsis thaliana. Plant and Cell Physiology 50: 956-969

Chuartzman SG, Nevo R, Shimoni E, Charuvi D, Kiss V, Ohad I, Brumfeld V, Reich Z (2008) Thylakoid membrane remodeling during state transitions in Arabidopsis. Plant Cell 20: 1029-1039

Daum B, Kuhlbrandt W (2011) Electron tomography of plant thylakoid membranes. Journal of Experimental Botany 62: 2393-2402

Daum B, Nicastro D, II JA, McIntosh JR, Kuhlbrandt W (2010) Arrangement of Photosystem II and ATP Synthase in Chloroplast Membranes of Spinach and Pea. Plant Cell 22: 1299-1312

El-Kafafi ES, Karamoko M, Pignot-Paintrand I, Grunwald D, Mandaron P, Lerbs-Mache S, Falconet D (2008) Developmentally regulated association of plastid division protein FtsZ1 with thylakoid membranes in Arabidopsis thaliana. Biochemical Journal 409: 87-94

Fitzpatrick LM, Keegstra K (2001) A method for isolating a high yield of Arabidopsis chloroplasts capable of efficient import of precursor proteins. Plant J 27: 59-65

Forth D, Pyke KA (2006) The suffulta mutation in tomato reveals a novel method of plastid replication during fruit ripening. J Exp Bot 57: 1971-1979

Gao H, Sage TL, Osteryoung KW (2006) FZL, an FZO-like protein in plants, is a determinant of thylakoid and chloroplast morphology. Proc Natl Acad Sci U S A 103: 6759-6764

Glynn JM, Froehlich JE, Osteryoung KW (2008) Arabidopsis ARC6 coordinates the division machineries of the inner and outer chloroplast membranes through interaction with PDV2 in the intermembrane space. Plant Cell 20: 2460-2470

Heslopharrison J (1963) Structure and morphogenesis of lamellar systems in grana-containing chloroplasts. Planta 60: 243-260

Heslopharrison J (1963) Structure and Morphogenesis of Lamellar Systems in Grana-Containing Chloroplasts .1. Membrane Structure and Lamellar Architecture. Planta 60: 243-260

Hinnah SC, Wagner R (1998) Thylakoid membranes contain a high-conductance channel. European Journal of Biochemistry 253: 606-613 
Junge W, Witt HT (1968) On Ion Transport System of Photosynthesis - Investigations on a Molecular Level. Zeitschrift Fur Naturforschung Part B-Chemie Biochemie Biophysik Biologie Und Verwandten Gebiete B 23: 244-\&

Karamoko M, El-Kafafi ES, Mandaron P, Lerbs-Mache S, Falconet D (2011) Multiple FtsZ2 isoforms involved in chloroplast division and biogenesis are developmentally associated with thylakoid membranes in Arabidopsis. Febs Letters 585: 1203-1208

Kroll D, Meierhoff K, Bechtold N, Kinoshita M, Westphal S, Vothknecht UC, Soll J, Westhoff P (2001) VIPP1, a nuclear gene of Arabidopsis thaliana essential for thylakoid membrane formation. Proc Natl Acad Sci U S A 98: 4238-4242

Kulandaivelu G, Gnanam A (1985) Scanning Electron-Microscopic Evidence for a Budding Mode of Chloroplast Multiplication in Higher-Plants. Physiologia Plantarum 63: 299-302

Leech RM, Thomson WW, Plattaloia KA (1981) Observations on the Mechanism of Chloroplast Division in Higher-Plants. New Phytologist 87: 1-\&

Lo SM, Theg SM (2012) Role of Vesicle-Inducing Protein in Plastids 1 in cpTat transport at the thylakoid. Plant J

Maple-Grodem J, Raynaud C (2014) Plastid Division. In SM Theg, FA Wollman, eds, Plastid Biology. Springer, New York, pp 155-188

McAndrew RS, Froehlich JE, Vitha S, Stokes KD, Osteryoung KW (2001) Colocalization of plastid division proteins in the chloroplast stromal compartment establishes a new functional relationship between FtsZ1 and FtsZ2 in higher plants. Plant Physiology 127: 1656-1666

Mercer FV, Hodge, A.J., Hope, A.B., Mclean, J. D. (1954) The Structure and Swelling Properties of Nitella Chloroplasts. Australian Journal of Biological Sciences 8: 1-18

Miyagishima SY, Froehlich JE, Osteryoung KW (2006) PDV1 and PDV2 mediate recruitment of the dynamin-related protein ARC5 to the plastid division site. Plant Cell 18: 2517-2530

Mustardy L, Buttle K, Steinbach G, Garab G (2008) The Three-Dimensional Network of the Thylakoid Membranes in Plants: Quasihelical Model of the Granum-Stroma Assembly. Plant Cell 20: 25522557

Mustardy LA, Janossy AGS (1979) Evidence of helical thylakoid arrangement by scanning electron microscopy. Plant Sci. Lett. 16: 281-284

Nierzwicki-Bauer SA, Balkwill DL, Stevens SE, Jr. (1983) Three-dimensional ultrastructure of a unicellular cyanobacterium. J Cell Biol 97: 713-722

Nishio JN, Whitmarsh J (1991) Dissipation of the Proton Electrochemical Potential in Intact and Lysed Chloroplasts .1. The Electrical Potential. Plant Physiology 95: 522-528

Oross JW, Possingham JV (1989) Ultrastructural Features of the Constricted Region of Dividing Plastids. Protoplasma 150: 131-138

Osteryoung KW, Pyke KA (2014) Division and dynamic morphology of plastids. Annu Rev Plant Biol 65: 443-472

Paolillo D, Falk R (1966) The ultrastructure of grana in mesolhyll plastids of Zea mays. Am J Botany 53: 173-180

Pyke KA, Leech RM (1994) A Genetic-Analysis of Chloroplast Division and Expansion in ArabidopsisThaliana. Plant Physiology 104: 201-207

Pyke KA, Rutherford SM, Robertson EJ, Leech RM (1994) Arc6, a Fertile Arabidopsis Mutant with Only 2 Mesophyll Cell Chloroplasts. Plant Physiology 106: 1169-1177

Robertson EJ, Pyke KA, Leech RM (1995) Arc6, an Extreme Chloroplast Division Mutant of Arabidopsis Also Alters Proplastid Proliferation and Morphology in Shoot and Root Apices. Journal of Cell Science 108: 2937-2944

Robertson EJ, Rutherford SM, Leech RM (1996) Characterization of chloroplast division using the Arabidopsis mutant arc5. Plant Physiology 112: 149-159 
Schoenknecht G, Althoff G, Junge W (1990) The electric unit size of thylakoid membranes. FEBS Lett. 277: $65-68$

Schoenknecht G, Althoff G, Junge W (1992) Dimerization constant and single-channel conductance of gramicidin in thylakoid membranes. Journal of Membrane Biology 126: 265-275

Shimoni E, Rav-Hon O, Ohad I, Brumfeld V, Reich Z (2005) Three-dimensional organization of higherplant chloroplast thylakoid membranes revealed by electron tomography. Plant Cell 17: 25802586

Theg SM, Tom C (2011) Measurement of the DeltapH and electric field developed across Arabidopsis thylakoids in the light. Methods Mol Biol 775: 327-341

van Roon H, van Breemen JFL, de Weerd FL, Dekker JP, Boekema EJ (2000) Solubilization of green plant thylakoid membranes with $\mathrm{n}$-dodecyl-alpha,D-maltoside. Implications for the structural organization of the Photosystem II, Photosystem I, ATP synthase and cytochrome b(6)f complexes. Photosynthesis Research 64: 155-166

Vitha S, Froehlich JE, Koksharova O, Pyke KA, van Erp H, Osteryoung KW (2003) ARC6 is a J-domain plastid division protein and an evolutionary descendant of the cyanobacterial cell division protein Ftn2. Plant Cell 15: 1918-1933

Wang Q, Sullivan RW, Kight A, Henry RL, Huang JR, Jones AM, Korth KL (2004) Deletion of the chloroplast-localized Thylakoid formation1 gene product in Arabidopsis leads to deficient thylakoid formation and variegated leaves. Plant Physiology 136: 3594-3604

Weier TE, Stocking CR, Bracker CE, Risley EB (1965) Structural Relationships of Internal Membrane Systems of in Situ and Isolated Chloroplasts of Hordeum Vulgare. American Journal of Botany 52: 339-\&

Whatley JM (1980) Plastid Growth and Division in Phaseolus-Vulgaris. New Phytologist 86: 1-\&

Witt HT (1979) Energy-Conversion in the Functional Membrane of Photosynthesis - Analysis by LightPulse and Electric Pulse Methods - Central Role of the Electric-Field. Biochimica Et Biophysica Acta 505: 355-427

Yoshida Y, Kuroiwa H, Misumi O, Nishida K, Yagisawa F, Fujiwara T, Nanamiya H, Kawamura F, Kuroiwa T (2006) Isolated chloroplast division machinery can actively constrict after stretching. Science 313: 1435-1438

Zhang M, Schmitz AJ, Kadirjan-Kalbach DK, Terbush AD, Osteryoung KW (2013) Chloroplast division protein ARC3 regulates chloroplast FtsZ-ring assembly and positioning in arabidopsis through interaction with FtsZ2. Plant Cell 25: 1787-1802 